\title{
Effect of disseminated intravascular coagulation on donation after citizens' death donor kidneys
}

\author{
Lu Xu, Wei Yin, Guanghui Li, Jiali Fang, Lei Zhang, Luhao Liu, Yunyi Xiong, Jialin Wu, Guanghui Pan, \\ Junjie Ma, Zheng Chen
}

Organ Transplantation Center of the Second Affiliated Hospital of Guangzhou Medical University, Guangzhou, China

Contributions: (I) Conception and design: L Xu; (II) Administrative support: G Pan, J Ma, Z Chen; (III) Provision of study materials or patients: W Yin, G Li, J Fang, L Zhang; (IV) Collection and assembly of data: L Xu, W Yin, J Wu; (V) Data analysis and interpretation: L Xu, L Liu, Y Xiong; (VI) Manuscript writing: All authors; (VII) Final approval of manuscript: All authors.

Correspondence to: Zheng Chen. Organ Transplantation Center of the Second Affiliated Hospital of Guangzhou Medical University, Guangzhou 510260, China. Email: docchenzheng@163.com.

Background: To investigate the effect of disseminated intravascular coagulation (DIC) on donor kidney in donation after citizens' death (DCD) donors.

Methods: The clinical and laboratory data of 159 DCD donors obtained by our center in 2018 were retrospectively analyzed. The DIC diagnosis was performed according to the Chinese DIC scoring system (CDSS). The donors were divided into two groups: DIC (+) and DIC (-). The difference between kidney rejection rate and zero puncture glomerular microthrombus formation rate were compared.

Results: Among the 159 DCD donors, 11 were discarded (accounting for $6.91 \%$ ). The reasons for the discarded cases included 5 cases $(3.14 \%)$ for moderate and severe glomerular microthrombus formation in the renal zero puncture pathology; 2 cases (1.26\%) for glomerular sclerosis ratio over 50\%; 2 cases $(1.26 \%)$ for long-term low blood pressure before pregnancy and significantly increased serum creatinine level and no urine; 1 case $(0.73 \%)$ for kidney stones and stagnant water; 1 case $(0.63 \%)$ for malignant tumor. The donor rejection rate of the DIC (+) group was higher than that of the DIC (-) group, and the difference was statistically significant $(\mathrm{P}<0.05)$. Among all donors, 10 cases $(6.29 \%)$ were found to have glomerular microthrombus at zero puncture, and the microthrombotic rate in the DIC (+) group was significantly higher than that in the DIC $(-)$ group $(\mathrm{P}<0.05)$. Of the 10 microthrombotic donors, 5 donors with severe glomerular microthrombus were discarded.

Conclusions: Donor-induced DIC can easily cause renal glomerular microthrombus formation, and the donor kidney rejection rate has increased.

Keywords: Intravascular coagulation donation after citizens' death donor kidney (DIC DCD donor kidney); discarded donor kidney; donor kidney microthrombus

Submitted Sep 27, 2019. Accepted for publication Jan 06, 2021.

doi: $10.21037 /$ tau-20-785

View this article at: http://dx.doi.org/10.21037/tau-20-785

\section{Introduction}

Donation after citizens' death (DCD) has become the main source of organ transplant donors (1). Timely and accurate assessment and maintenance of DCD donors are key factors in ensuring the function of organ donation, obtaining more effective organs, and achieving better transplant outcomes (2). Disseminated intravascular coagulation
(DIC) can cause multiple organ dysfunction syndrome (MODS), leading to renal microthrombus and causing renal failure; preventing and treating DCD donor DIC in the early stage can thus improve organ utilization $(3,4)$. This study reviewed 159 DCD donors from January 1, 2018 to December 31, 2018 in our center to investigate the effects of DIC on kidney supply. 
Table 1 General information on donors

\begin{tabular}{lccc}
\hline Characteristics & Total number of donors, $\mathrm{n}=159$ & DIC (+) donor, $\mathrm{n}=50$ & DIC $(-)$ donor, $\mathrm{n}=109$ \\
\hline Median age & $40[2-64]$ & $41[2-62]$ & 39 [2-64] \\
Gender (male/female) & $144 / 15$ & $47 / 3$ & $97 / 12$ \\
Primary disease & & 33 & 51 \\
Brain trauma & 84 & 11 & 47 \\
Intracranial bleeding & 58 & 1 & 6 \\
Brain tumor & 7 & 3 & 1 \\
Hypoxic brain injury & 4 & 2 & 4 \\
Others & 6 & 36 & 16 \\
Chinese classification & 129 & 14 & 93 \\
I & 30 & 3 & 16 \\
\hline
\end{tabular}

Other primary diseases include poisoning and Japanese encephalitis. DIC, disseminated intravascular coagulation.

We present the following article in accordance with the STROBE reporting checklist (available at http://dx.doi. org/10.21037/tau-20-785).

\section{Methods}

\section{General information}

We summarized the data from 159 DCD donors from January 1, 2018 to December 31, 2018, including 144 males and 15 females. They were divided into the DIC (+) and DIC (-) groups (Table 1), and the difference between the two groups in donor kidney rejection rate and zero puncture glomerular microthrombus formation rate was determined. The donor was diagnosed with DIC according to the Chinese DIC scoring system (CDSS).

\section{Ethic}

All procedures performed in this study involving human participants were in accordance with the Declaration of Helsinki (as revised in 2013). The study was approved by the ethics committee of the Second Affiliated Hospital of Guangzhou Medical University and the number of the approval was (No. 2015025). Individual consent for this retrospective analysis was waived.

\section{Statistical analysis}

Statistical analysis was performed using SPSS 22.0 software. Pearson chi-square test was used for the renal deprivation rate. The glomerular microthrombus formation rate was determined by continuous calibration chi-square test. $\mathrm{P}$ was less than 0.05 , and the difference was statistically significant.

\section{Results}

Among the 159 donors, 50 were diagnosed with DIC $(31.45 \%)$ and 11 were excluded (6.91\%). The excluded cases consisted of the following: 5 cases $(3.14 \%)$ for moderate and severe glomerular microthrombus formation in the renal zero puncture pathology; 2 cases (1.26\%) for glomerular sclerosis ratio over $50 \% ; 2$ cases $(1.26 \%)$ for long-term low blood pressure before pregnancy and significantly increased serum creatinine level and no urine; 1 case $(0.73 \%)$ for kidney stones and stagnant water; 1 case $(0.63 \%)$ for malignant tumor. The donor rejection rate of the DIC $(+)$ group was higher than that of the DIC (-) group, and the difference was statistically significant $(\mathrm{P}<0.05)$. Among all donors, 10 cases $(6.29 \%)$ were found to have glomerular microthrombus at zero puncture, and the microthrombotic rate in the DIC (+) group was significantly higher than that in the DIC $(-)$ group $(\mathrm{P}<0.05)($ Table 2$)$. Of the 10 
Table 2 Comparison of the donor kidney rejection rate and microthrombus formation rate between the DIC (+) and DIC (-) groups

\begin{tabular}{lccc}
\hline Groups & Total number of donors, $\mathrm{n}=159$ & DIC (+) donor, $\mathrm{n}=50$ & DIC (-) donor, $\mathrm{n}=109$ \\
\hline Kidney abandonment & $11(6.91 \%)$ & $7(14.00 \%)$ & $4(3.67 \%)$ \\
Glomerular microthrombus & $10(6.29 \%)$ & $9(18.00)$ & $1(0.91 \%)$ \\
\hline
\end{tabular}

DIC, disseminated intravascular coagulation.

microthrombotic donors, 5 donors with severe glomerular microthrombus were discarded, and the other 5 cases were mild microthrombus formation, which was successfully used for transplantation.

\section{Discussion}

\section{The definition, diagnosis, and the cause of DIC}

\section{Definition of DIC}

DIC is defined as an acquired syndrome characterized by a localized intravascular coagulation system activation arising from different causes including damage to the microvascular system and the microvascular system during the progression process, resulting in a vicious cycle, with serious damage possibly leading to MODS (2). Some researchers believe that DIC should be understood as diffuse intravascular microthrombosis (DIT) associated with vascular endothelial cell disease, which can basically explain the clinical features of all DIC (3).

\section{Diagnosis of DIC}

It is necessary to combine basic diseases, clinical manifestations, and laboratory indicators. In 1986, the diagnostic criteria for DIC were first proposed in China, and it was revised several times (4). The revised Chinese Expert Consensus for Diagnosis and Treatment of Disseminated Intravascular Coagulation in 2012 is a diagnostic standard widely accepted and applied by clinicians in China, but it still has deficiencies. International experts from Europe, America, and Japan have developed a multi-index DIC score diagnosis system which comprises the International Thrombosis and Hemostasis Association Standard (ISTH) $(5,6)$, the Japanese Ministry of Health and Welfare Standard (JMHW), the Japanese Emergency Medicine Society Standard (JAAM), and the Japan Thrombosis and Hemostasis Association (JSTH) standards (7-10). However, the integral system above is currently confusing for clinical use in China, and there is no research data confirming the integral system in the Chinese population. Since 2014, the Chinese Medical Association Blood Branch Thrombus and
Hemostasis Group has established a Chinese Disseminated Intravascular Coagulation System (CDSS) through a large number of studies, and published the Disseminated Intravascular Coagulation Diagnostics in 2017. The Chinese Expert Consensus (2017 Edition) makes the DIC diagnostic standards more in line with China's national conditions (11). The Affiliated Union Hospital of Tongji Medical College of Huazhong University of Science and Technology conducted a retrospective data analysis on 629 patients with suspected DIC in the hospital and found that CDSS has good diagnostic performance: it is simple and practical, and easy to be promoted clinically (12). Wu et al. conducted a prospective clinical study on 753 suspected DIC patients in 18 centers across the country to compare CDSS, ISTH, JAAM, and JMHW score systems in DIC diagnosis and 28-day mortality prediction. The results showed that CDSS was superior to ISTH. CDSS was superior to JAAM, and, in comparison with ISTH, it was also superior to JHMW. Taken together, this proves that the CDSS integral system has higher sensitivity and specificity, thus supporting the use of CDSS to predict the severity and prognosis of the disease (13).

\section{Causes of DIC}

(I) Infectious diseases are the most important and common cause. Serious infections of various pathogens can cause DIC. The DCD donors from the intensive care unit are prone to serious infections of various resistant bacteria, which can significantly increase the possibility of DIC.

(II) Malignant tumor causes include acute leukemia, lymphoma, pancreatic cancer, liver cancer, prostate cancer, and kidney cancer.

(III) Pathology obstetrics is also one of the most important causes for obstetric bleeding and maternal death.

(IV) Surgery and trauma can promote the brain, prostate, pancreas and other organs to release a significant amount of tissue factor, leading to DIC. Severe crush injuries, severe fractures, and extensive burns can 
also cause DIC. Craniocerebral injury is the most common cause of DCD donors, and some brain surgery can cause DIC to a large degree.

(V) Other causes include heatstroke, ARDS, acute intravascular hemolysis, acute pancreatitis, and malignant hypertension.

\section{The impact of DIC on the kidney}

DCD has become the main source of organ transplant donors, which can effectively alleviate the pressure of a serious donor shortage (1). However, the quality of DCD donors is unstable, and organ maintenance function is directly related to donation success rate and recipient safety. The main measures include monitoring invasive arterial pressure, central venous pressure, heart rate, body temperature, urine output, testing DIC, blood gas analysis, electrolyte, and acid-base balance, blood routine, urine routine, and liver and kidney function. According to the monitoring results, effective measures are taken to maintain the blood perfusion and function of donor organs (14).

For many diseases, DIC is an intermediate link in the pathological process. The main clinical manifestations are severe bleeding, microcirculatory dysfunction, microvascular embolism, and microvascular hemolysis (4). Most DIC patients have rapid onset, complicated condition, rapid development, and prognosis. If they are not diagnosed and treated in time, they often endanger the life of the DCD donor, which makes the organ acquisition process uncontrollable while not being conducive to organ function maintenance. At the same time, DIC induces MODS, causing liver and kidney failure, and activation of the systemic coagulation system, resulting in the formation of intravascular fibrin microthrombus. Intrarenal microthrombi can form in the kidney, as it has one of the richest supplies of blood in the body (15). At the same time, the fibrinolytic system activates to form a large amount of plasmin, which degrades fibrin and produces a wide range of fibrin (-ogen) degradation products (FDP), which in turn block the renal capillaries and impair renal perfusion. FDP also enhances the action of histamine and kinins, promotes microcirculation and blood stasis, and triggers the accumulation of red blood cells, platelets, and leukocytes in capillaries (16). The activation of the coagulation and fibrinolysis system is disordered, releasing a large number of active factors, further impairing the endocrine function of endothelial cells, causing the production and imbalance of endothelin (the vasoactive factor) and nitric oxide (the vasodilator factor), leading to intrarenal vasoconstriction and thus a continuous low myeloid perfusion (17). The factors above aggravate renal damage.

DIC is one of the common causes for donor kidney abandonment (18). In this study, the donor rejection rate and microthrombotic rate in the DIC (+) group were significantly higher than those in the DIC (-) group, while DIC prevention and treatment is important for maintaining renal function. However, DIC and microthrombus formation are not taboos for organ donation. In this study, there were 9 cases of DIC (+) donors with microthrombus, and 1 case of DIC (-) donors showing microthrombus, which could have been non-dominant DIC (19). The score failed to meet the diagnostic criteria of DIC, or there were traumatic coagulopathy (20). Among the 10 microthrombotic donors, there were still 5 donors with bilateral donor kidneys which were used for transplantation. Because of the zero puncture pathology, the microthrombus was mild, and was probably related to the fact that we immediately use heparin and blood transfusion (21-23) and acquire organs as soon as possible; there are similar reports abroad (24).

In order to ensure the quality of donor organs, the medical level for potential donor discovery and maintenance must be improved. The detection and treatment of DIC in an early stage, and harvesting the organ as soon as possible, are critical in minimizing the adverse factors affecting organ function (25). Furthermore, the rapid-freezing pathological examination on the kidney zero puncture is important for assessing whether the donor kidney should be abandoned. The LifePort renal transporter can also be used for renal perfusion, according to perfusion flow and resistance index, while the quality of the donor kidney can be quickly and effectively judged (26), and even improved, by LifePort perfusion (27).

\section{Acknowledgments}

Funding: None.

\section{Footnote}

Reporting Checklist: The authors have completed the STROBE reporting checklist. Available at http://dx.doi. org/10.21037/tau-20-785

Data Sharing Statement: Available at http://dx.doi. 
org/10.21037/tau-20-785

Conflicts of Interest: All authors have completed the ICMJE uniform disclosure form (available at http://dx.doi. org/10.21037/tau-20-785). The authors have no conflicts of interest to declare.

Ethical Statement: The authors are accountable for all aspects of the work in ensuring that questions related to the accuracy or integrity of any part of the work are appropriately investigated and resolved. All procedures performed in this study involving human participants were in accordance with the Declaration of Helsinki (as revised in 2013). The study was approved by the ethics committee of the Second Affiliated Hospital of Guangzhou Medical University and the number of the approval was (No. 2015025). Individual consent for this retrospective analysis was waived.

Open Access Statement: This is an Open Access article distributed in accordance with the Creative Commons Attribution-NonCommercial-NoDerivs 4.0 International License (CC BY-NC-ND 4.0), which permits the noncommercial replication and distribution of the article with the strict proviso that no changes or edits are made and the original work is properly cited (including links to both the formal publication through the relevant DOI and the license). See: https://creativecommons.org/licenses/by-nc-nd/4.0/.

\section{References}

1. Huang J, Millis JM, Mao Y, et al. Voluntary organ donation system adapted to Chines cultural values and social reality. Liver Transpl 2015;21:419-22.

2. Thachil J. Disseminated intravascular coagulation-new pathophysiological concepts and impact on management. Expert Rev Hematol 2016;9:803-14.

3. Chang JC. Disseminated intravascular coagulation: is it fact or fancy? Blood Coagul Fibrinolysis 2018;29:330-7.

4. Song S, Wang H, Li J. Disseminated intravascular coagulation M. Second edition, Shanghai: Shanghai Science and Technology Press 2001:146-66.

5. Taylor FB Jr, Toh CH, Hoots WK, et al. Towards definition, clinical and laboratory criteria, and a scoring system for disseminated intravascular coagulation. Thromb Haemost 2001;86:1327-30.

6. Di Nisio M, Baudo F, Cosmi B, et al. Diagnosis and treatment of disseminated intravascular coagulation:guidelines of the Italian Society for Haemostasis and Thrombosis(SISET). Thromb Res 2012;129:e177-84.

7. Wada H, Thachil J, Di Nisio M, et al. Guidance for diagnosis and treatment of DIC from harmonization of the recommendations from three guidelines. J Thromb Haemost 2013. [Epub ahead of print]. doi: 10.1111/ jth.12155.

8. Wada H, Takahashi H, Uchiyama T, et al. The approval of revised diagnostic criteria for DIC from the Japanese Society on Thrombosis and Hemostasis. Thromb J 2017;15:17.

9. Gando S, Saitoh D, Ogura H, et al. Natural history of disseminated intravascular coagulation diagnosed based on the newly established diagnostic criteria for critically ill patients: results of a multicenter, prospective survey. Crit Care Med 2008;36:145-50.

10. Takemitsu T, Wada H, Hatada T, et al. Prospective evaluation of three different diagnostic criteria for disseminated intravascular coagulation. Thromb Haemost 2011;105:40-4.

11. Chinese Medical Association Hematology Branch Thrombosis and Hemostasis Group. Disseminated intravascular coagulation diagnosis Chinese expert consensus (2017 edition). Chinese Journal of Hematology 2017;38:361-3.

12. Wang M, Kou H, Deng J, et al. Retrospective Evaluation of New Chinese Diagnostic Scoring System for Disseminated Intravascular Coagulation. PLos One 2015;10:e0129170.

13. Wu Y, Luo L, Niu T, et al. Evaluation of the new Chinese Disseminated Intravascular Coagulation Scoring System in critically ill patients: A multicenter prospective study. J Sci Rep 201;7:9057.

14. Black CK, Termanini KM, Aguirre O, et al. Solid organ transplantation in the 21st century. Ann Transl Med 2018;6:409.

15. Clermont G, Acker CG, Angus DC, et al. Renal failure in the ICU: Comparison of the impact of acute reanl failure and endstage renal disease on ICU outcomes. Kidney Int 2002;62:986-96.

16. Wada $\mathrm{H}$, Wakita $\mathrm{Y}$, Nakase $\mathrm{T}$, et al. Increased plasmasoluble fibrin monomer levels in patients with disseminated intravascular coagulation. Am J Hematol 1996;51:255-60.

17. Knotek M, Rogachev B, Wang W, et al. Endotoxemic renal failure in mice: role of tumor necrosis factor independent of inducible nitric oxide synthase. Kidney Int 2001;59:2243-9. 
18. Wang CJ, Shafique S, McCullagh J, et al, Implications of donor disseminated intravascular coagulation on kidney allograft recipients. Clin J Am Soc Nephrol 2011;6:1160-7.

19. Squizzato A, Rancan E, Thachil J, et al. Diagnosis of overt and non-overt disseminated intravascular coagulation: A survey among experts and a call for action from the ISTH. Thromb Res 2017;152:74-6.

20. Davenport RA, Guerreiro M, Frith D, et al. Activated Protein C Drives the Hyperfibrinolysis of Acute Traumatic Coagulopathy. Anesthesiology 2017;126:115.

21. Selighsohn U. Eisseminated intravascular coagulation. In: Beutler E, Lichtman MA, Coller BS, et al. editors. Williams hematology. 6th ed. New York: McGraw-Hill, 2001:1677-95.

22. Kvasnicka J, Ehler Z, Polivkova J, et al. Disseminated intravascular coagulation syndrome and protein C. Sb Lek 2002;103:257.

23. Sugawara Y. Living-donor liver transplantation for

Cite this article as: $\mathrm{Xu} \mathrm{L}$, Yin W, Li G, Fang J, Zhang L, Liu L, Xiong Y, Wu J, Pan G, Ma J, Chen Z. Effect of disseminated intravascular coagulation on donation after citizens' death donor kidneys. Transl Androl Urol 2021;10(3):1273-1278. doi: 10.21037/tau-20-785 hepatocellular carcinoma in Japan and Korea. Transl Cancer Res 2017;6:S1409-11.

24. Revollo JY, Cuffy MC, Witte DP, et al. Case Report: Hemolytic Anemia Following Deceased Donor Renal Transplantation Associated With Tranexamic Acid Administration for Disseminated Intravascular Coagulation. Transplant Proc 2015;47:2239-42.

25. Fu Xi, Wang Changxi, Li Jun, et al. Clinical analysis on 233 cases of organ donation kidney transplantation after the death of citizens. Chinese Journal of Urology 2014;35:15-9.

26. Patel SK, Pankewycz OG, Nader ND, et al. Prognostic utility of hypothermic machine perfusion in deceased donor renal transplanration. Transplant Proc 2012;44:2207-12.

27. Hosgood SA, Yang B, Bagul A, et al. A comparison of hypothermic machine perfusion versus static cold storage in an experimental model of renal ischemia reperfusion injury. Transplantation 2010;89:830-7. 